\title{
Effects of scan circle displacement in optical coherence tomography retinal nerve fibre layer thickness measurement: a RNFL modelling study
}

${ }^{1}$ Department of Ophthalmology and Visual Sciences, The Chinese University of Hong Kong, Hong Kong, PRC

${ }^{2}$ Department of Applied Mathematics, Hong Kong Polytechnic University, Hong Kong, PRC

${ }^{3}$ Hamilton Glaucoma Center, Department of Ophthalmology, University of California, San Diego, CA, USA

Correspondence:

CKS Leung,

Department of

Ophthalmology \& Visual

Sciences,

The Chinese University of Hong Kong,

Hong Kong Eye Hospital, 147K Argyle Street, Kowloon, Hong Kong

Tel: + 852 27623080;

Fax: + 85227159490 .

E-mail: tlims00@

hotmail.com

Received: 7 November 2007 Accepted in revised form: 17 July 2008

Published online: 19

September 2008

\begin{abstract}
Objective To study the effect of optical coherence tomography (OCT) scan circle displacement on retinal nerve fibre layer (RNFL) measurement errors using cubic spline models. Methods Forty-nine normal subjects were included in the analysis. In one randomly selected eye in each subject, RNFL thickness around the optic disc was measured by taking 16 circular scans of different sizes (scan radius ranged from 1 to $2.5 \mathrm{~mm}$ ). The RNFL profile in each eye was constructed with a mathematical model using a smoothing spline

approximation. Scan circle (diameter $3.4 \mathrm{~mm}$ ) RNFL measurements (total average, superior, nasal, inferior, and temporal RNFL thicknesses) obtained from eight directions (superior, superonasal, nasal, inferonasal, inferior, inferotemporal, temporal, and superotemporal) displaced at different distances $(0.1,0.2,0.3,0.4,0.5,0.6$, and $0.7 \mathrm{~mm})$ from the disc centre were then computed by a computer program and compared to the 'reference standard' where the scan circle is centred at the optic disc. RNFL measurement error was calculated as the absolute of (RNFL thickness (displaced) - RNFL thickness (reference standard).

Results The respective mean average, superior, nasal, inferior, and temporal RNFL measurement errors were $2.3 \pm 2.0,4.9 \pm 4.5$, $4.1 \pm 3.8,6.2 \pm 7.6$, and $3.8 \pm 3.5 \mu \mathrm{m}$ upon $0.1 \mathrm{~mm}$ scan circle displacement, and $12.1 \pm 11.4$, $27.8 \pm 18.4,21.7 \pm 18.6,34.8 \pm 22.9$, and $15.2 \pm 10.7 \mu \mathrm{m}$ upon $0.7 \mathrm{~mm}$ scan circle
\end{abstract}

CYL Cheung ${ }^{1}$, CKF Yiü ${ }^{2}$ RN Weinreb ${ }^{3}$, D Lin', $\mathrm{H} \mathrm{Li}^{1}, \mathrm{AY}$ Yung ${ }^{1}, \mathrm{CP}$ Pang ${ }^{1}$, DSC Lam ${ }^{1}$ and CKS Leung ${ }^{1,3}$ displacement. Significant differences of average and quadrant RNFL thicknesses were evident between centred and displaced scan circle measurements (all with $P<0.001$ ). RNFL measurement error increased in a monotonic fashion with increasing distance away from the disc and the change was directiondependent.

Conclusions RNFL measurement error varies with the direction and distance of scan displacement. The superior and the inferior RNFL measurements are most vulnerable to scan displacement errors, whereas the average RNFL thickness is the least susceptible. Obtaining a well-centred scan is essential for reliable RNFL measurement in OCT. Eye (2009) 23, 1436-1441; doi:10.1038/eye.2008.258; published online 19 September 2008

Keywords: RNFL; OCT; scan circle displacement; cubic spline model; 3D RNFL profile; RNFL measurement error

Introduction

Assessment of retinal nerve fibre layer (RNFL) is important in the diagnosis and monitoring of glaucoma. Optical coherence tomography (OCT) is one of the imaging instruments that has been shown to provide reproducible measurement of RNFL thickness ${ }^{1-3}$ and found to be useful in detecting glaucomatous change. ${ }^{4-7}$ In the default setting, OCT measures RNFL thickness using a circular scan with a 
diameter of $3.4 \mathrm{~mm}$ centred at the optic disc.

The mean of three sequential circular scans is obtained and compared with the normative database. To obtain reliable and reproducible RNFL thickness, it is mandatory to position the centre of the scan circle at the centre of the optic disc. However, apart from viewing the scan circle in the fundus photograph taken by the realtime charge-couple device, there is no objective index to indicate the centrality of the scan circle. It is conceivable that the displacement of the scan circle from the optic disc centre would result in different values of RNFL thickness and lead to potential diagnostic errors. Inconsistent scan circle position also adds 'noise' during serial analysis and thus reducing the sensitivity and specificity to detect change.

It is unknown, however, to what extent the RNFL measurements would be affected by scan circle displacement because it is not possible to measure how much the scan is displaced relative to the optic disc in the OCT. The purpose of this study is to develop a model that can be used to predict and estimate the change in RNFL profiles when the scan circle is displaced at different directions and distances away from the optic disc centre. This information could be useful to estimate the magnitude of measurement error in OCT RNFL scanning and to determine the limits of measurement variability during serial analysis of RNFL.

\section{Methods}

\section{Subjects}

Fifty-three healthy normal volunteers were recruited at the University Eye Center, The Chinese University of Hong Kong. All subjects underwent ophthalmic examination including visual acuity, refraction, intraocular pressure measurement, and refraction. One eye was then selected in random for visual field test and OCT RNFL measurement (see below). Other than refractive error, all included eyes had normal visual field results, with best-corrected visual acuity of at least 20/40 and without concurrent ocular pathology. Four subjects who did not have steady fixation were excluded. The study was conducted in accordance with the ethical standards stated in the Declaration of Helsinki and approved by the local clinical research ethics committee with informed consent obtained.

\section{Visual field test}

Standard visual field testing was performed with the static automated white-on-white threshold perimetry (Humphrey Field Analyser II; Humphrey Instruments, Dublin, CA, USA). A visual field was defined as reliable when fixation losses, false-positive, and false-negative rates were less than $25 \%$. A visual field defect was defined as having three or more significant $(P<0.05)$ nonedge contiguous points with at least one at the $P<0.01$ level on the same side of horizontal meridian in the pattern deviation plot and classified as outside normal limits in the glaucoma hemifield test. Visual field was performed to ensure that all the eyes included were normal and demonstrated no glaucomatous damage.

\section{Optical coherence tomography RNFL measurement}

Optical coherence tomography was performed with OCT version 3 (STRATUS OCT; Carl Zeiss Meditec Inc., Dublin, CA, USA). The 'circle' protocol (512 A-scans) was selected to collect concentric RNFL measurements around the optic disc. Each eye was imaged with 16 circular scans of different sizes, starting with scan radius of $1 \mathrm{~mm}$ with $0.1 \mathrm{~mm}$ increment in radius in subsequent scans (ie, scan radius ranged from 1 to $2.5 \mathrm{~mm}$ ). The 'repeat' mode was used to ensure that each scan is well-centred and to minimize adjustment of the scan position. All the OCT measurements were taken by an experienced operator with signal strength of at least 7 . In the OCT images, RNFL can be delineated and visualized as the first layer in high reflectivity signal. RNFL thickness is determined by the difference in distance between the vitreoretinal interface and a posterior border based on a predefined reflectivity signal level. The 16 images in each subject were exported for analysis for mathematical modelling.

\section{Mathematical modelling: construction of 3D RNFL profile}

As RNFL thickness data are available only in discrete circular scans, mathematical modelling was used to derive a close approximation of RNFL thickness values at the 'gaps' between concentric circles. A total of $512 \times 16$ RNFL thickness data points were collected in each eye for model fitting. Each data point $\left\{z\left(r_{\mathrm{i}}, \theta_{\mathrm{i}}\right): r 1 \leqslant r \mathrm{i} \leqslant r 2.5\right.$, $\left.0 \leqslant \theta_{\mathrm{i}} \leqslant 2 \pi\right\}$ has specific coordinates with reference to the optic disc centre as the origin. The cubic splines, which are piecewise cubic polynomial functions with continuous gradient and curvature, were used to derive the approximate surface. ${ }^{8}$ This provides a polynomial of a relatively low degree with good approximation to any smooth nonlinear functions. An error function is defined to be a mismatch between the spline surface and the measurements, plus a penalty term for the smoothness of the spline surface, as follows:

$$
\sum_{r_{i}} \sum_{\theta_{i}}\left(z\left(r_{i}, \theta_{i}\right)-S\left(r_{i}, \theta_{i}\right)\right)+\lambda \int\left|\nabla^{2} S\right| \mathrm{d} A
$$


By minimizing this error function, a smoothing spline approximation is obtained. Note that $S(r \mathrm{i}, \theta \mathrm{i})$ is a multivariate spline function obtained from univariate spline by the tensor product construct, and $\lambda$ is a smoothing parameter. Figure 1a illustrates an example of a 3D RNFL topology after model fitting.

\section{Calculation of scan circle displacement error with $3 D$ RNFL profiles}

On the basis of the derived 3D RNFL profile, a computer program was written using Matlab version 6.5 (The Math Works, Natick, MA, USA) to evaluate the circular RNFL measurements at specific direction and distance away from the optic disc centre. Scan circle (diameter $3.4 \mathrm{~mm}$ ) RNFL measurements (total average, superior, nasal, inferior, and temporal RNFL thicknesses) obtained from eight directions (superior, superonasal, nasal, inferonasal, inferior, inferotemporal, temporal, and superotemporal) displaced at different distances (0.1, 0.2 $0.3,0,4,0.5,0.6$, and $0.7 \mathrm{~mm}$ ) from the disc centre were computed by the program and compared with the 'reference standard' where the scan circle is centred at the optic disc. RNFL measurement errors were calculated as the absolute of (RNFL thickness (displaced) - RNFL thickness (reference standard). Mean RNFL measurement error at a particular displacement distance was calculated as the average of superior, nasal, inferior, and temporal RNFL measurement error measured at that particular displacement distance.

\section{Statistical analysis}

Statistical analyses were performed using SPSS version 11.0 (SPSS Inc., Chicago, IL, USA). The associations between RNFL measurements and displacement distance were evaluated with linear regression analysis and the correlations were expressed as Spearman correlation coefficients. $P<0.05$ was considered statistically significant. Repeated-measure analysis of variance was used for the comparisons of the mean RNFL measurement error at different scan displacement distances.

\section{Results}

Forty-nine normal eyes from 49 subjects were analysed in this study. The age ranged from 21 to 60 years with mean \pm SD of $35.2 \pm 11.7$ years. The average spherical error was $-1.9 \pm 2.3$ dioptres. The average, superior, nasal, inferior, and temporal RNFL thicknesses measured with the $3.4 \mathrm{~mm}$ scan circle centred at the optic disc (the reference standard) were 108.6 $\pm 10.9,133.3 \pm 16.3$, $70.7 \pm 16.8,138.5 \pm 18.8$, and $91.3 \pm 21.8 \mu \mathrm{m}$, respectively.

Figure 1 presents an example of changes in the RNFL profiles with respect to the change in direction and distance of scan circle placement. Although changes in the RNFL profile are already observed when the scan circle was displaced for only $0.1 \mathrm{~mm}$ (Figure $1 \mathrm{~b}-\mathrm{e}$, upper panel), the differences became more obvious when the scan circle was displaced at a longer distance away from the optic disc (Figure 1b-e, middle panel). Superior and inferior displacement in general resulted in more changes in the RNFL profiles compared with temporal and nasal displacement. The RNFL profiles with scan circle displaced at an arbitrary distance at four different directions (temporal, superior, nasal, and inferior) during OCT imaging are illustrated in the bottom panel. It was observed that the patterns of RNFL profiles (centred and displaced scans) were similar between the modelled profiles and those in the OCT serial analysis printouts.

The average and quadrant RNFL measurement errors (RNFL thickness (displaced) - RNFL thickness (reference) were calculated with reference to the distance and the direction of scan circle displacement (Tables 1 and 2). Irrespective of the direction of displacement, the average and quadrant RNFL measurement errors increased with the distance of displacement. The mean average RNFL measurement errors upon 0.1 and $0.7 \mathrm{~mm}$ scan circle displacement were $2.3 \pm 2.0$ and $12.1 \pm 11.4 \mu \mathrm{m}$, respectively. The respective mean superior, nasal, inferior, and temporal RNFL measurement errors were $4.9 \pm 4.5,4.1 \pm 3.8,6.2 \pm 7.6$, and $3.8 \pm 3.5 \mu \mathrm{m}$ upon $0.1 \mathrm{~mm}$ scan circle displacement, and $27.8 \pm 18.4,21.7 \pm 18.6$, $34.8 \pm 22.9$, and $15.2 \pm 10.7 \mu \mathrm{m}$ upon $0.7 \mathrm{~mm}$ scan circle displacement (Table 2). The superior and inferior RNFL measurements were most susceptible to scan circle displacement errors, whereas the average and the temporal RNFL measurements were the least susceptible.

\section{Discussion}

In this study, we showed that scan centration is critical for reliable measurement of RNFL thickness. Using cubic spline models, it is observed that the RNFL measurement error varied with the displacement distance and the displacement direction of the scan circle.

Optical coherence tomography measurement of RNFL thickness has been reported to be reproducible. Budenz DL et $a l^{1}$ showed that the intraclass correlation (ICC) for the mean RNFL 3.4 thickness (512 A-scans) was 0.97 and the coefficient of variation was $1.7 \%$. Gurses-Ozden et $a l^{2}$ reported the coefficient of variation for mean RNFL 3.4 thickness (512 A-scans) ranged between 7.3 and 8.0\%. It should be noted that in these studies, RNFL measurements were taken during the same session. In the study by Paunescu et $\mathrm{al}^{3}$ in which RNFL measurements were taken at three different sessions in different days within 5 months, the reproducibility was lower. ${ }^{3}$ The ICC for mean RNFL 3.4 thickness 


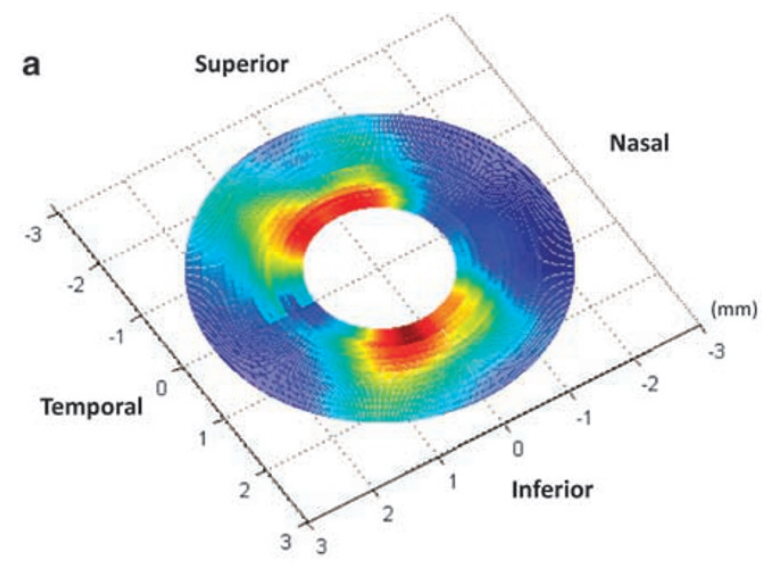

b
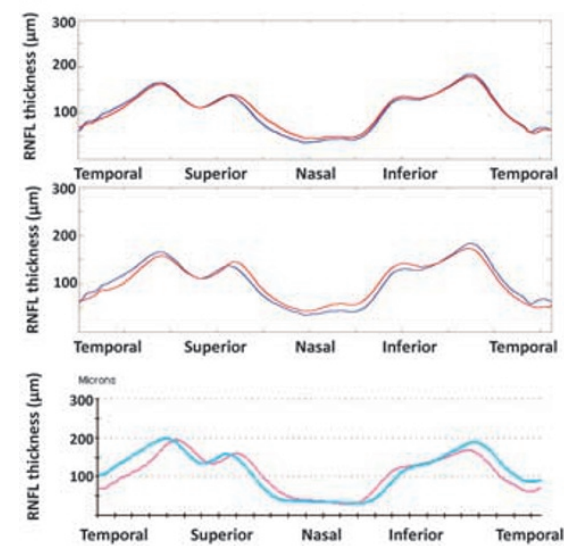

d
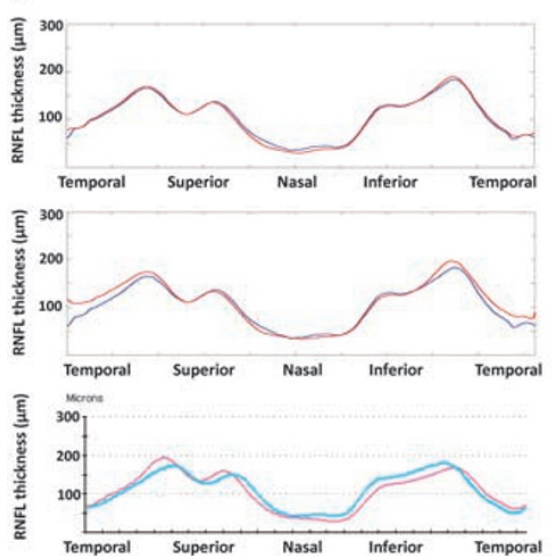
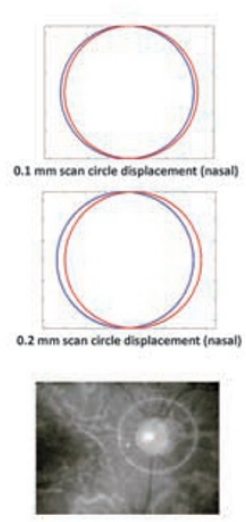

\section{c}

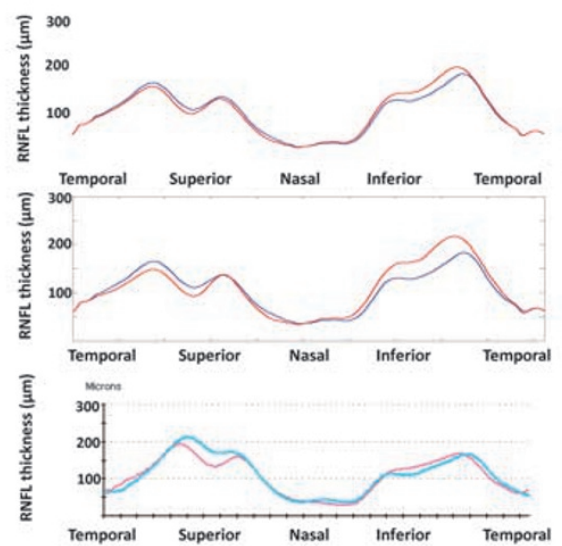

e
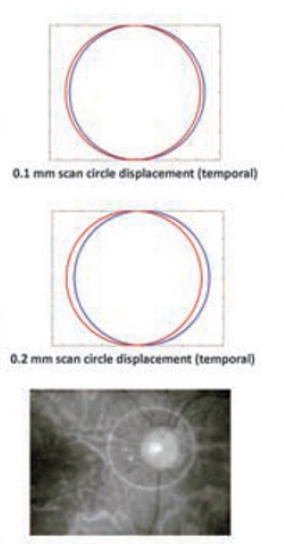
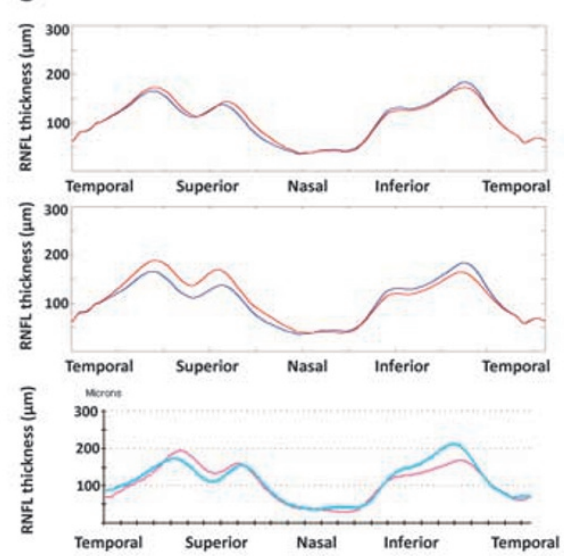
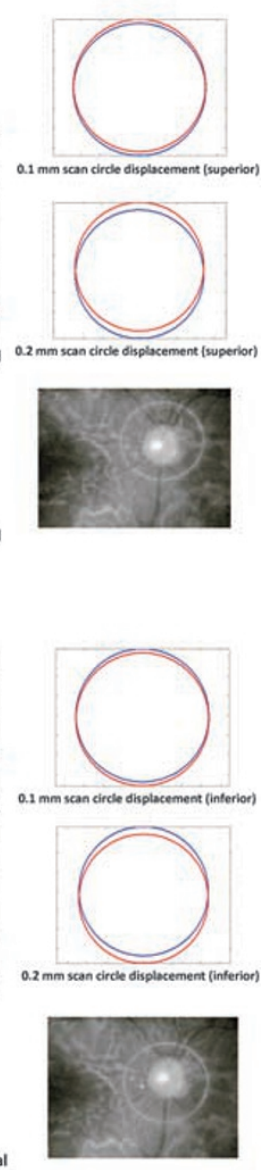

Figure 1 Retinal nerve fibre layer (RNFL) topology around the optic disc derived from a mathematical model using smoothing spline approximation based on measurements obtained from 16 OCT circular scans with different scan sizes (scan radius ranged from 1 to $2.5 \mathrm{~mm}$ ) in a normal eye (a). A false colour-encoding scale was applied with red indicating high RNFL thickness values and blue indicating low RNFL thickness values. The derived RNFL profiles with the scan circle displaced nasally (b), superiorly (c), temporally (d), and inferiorly (e) at a distance of $0.1 \mathrm{~mm}$ (upper panels) and $0.2 \mathrm{~mm}$ (middle panels) are shown in red. The reference RNFL profile is shown in blue. By displacing the scan circle at an arbitrary distance during OCT imaging, the RNFL profiles (centred and displaced scans) were obtained using the fast RNFL 3.4 scan protocol in the same eye and shown in the serial analysis printout in the bottom panel. The RNFL profile centred at the disc is shown in blue and those with scan circles displaced temporally (b), superiorly (c), nasally (d), and inferiorly (e) (shown in the corresponding fundus photographs) are in red. 
Table 1 Linear regression analyses of average/quadrants retinal nerve fibre layer (RNFL) measurement errors vs distance of scan displacement at different displacement directions

\begin{tabular}{llcc}
\hline $\begin{array}{l}\text { RNFL } \\
\text { measurement }\end{array}$ & $\begin{array}{l}\text { Displacement } \\
\text { direction }\end{array}$ & $\begin{array}{c}\text { Linear } \\
\text { regression }\end{array}$ & $\mathrm{r}$ \\
\hline Average & Superotemporal & $y=25.54 x+1.56$ & 0.678 \\
Superior & Inferotemporal & $y=62.76 x-2.00$ & 0.797 \\
Nasal & Superotemporal & $y=54.70 x-3.51$ & 0.762 \\
Inferior & Superotemporal & $y=92.28 x-3.83$ & 0.858 \\
Temporal & Temporal & $y=24.87 x+3.18$ & 0.616 \\
\hline
\end{tabular}

Only the regression models with the largest correlation coefficient in the average and each of the quadrant RNFL measurement are shown.

Table 2 Mean average and quadrant RNFL measurement errors at different scan displacement distances away from the optic disc centre

\begin{tabular}{|c|c|c|c|}
\hline $\begin{array}{l}\text { RNFL } \\
\text { measurement } \\
\text { error }\end{array}$ & $\begin{array}{l}\text { Scan } \\
\text { displacement } \\
\text { distance }(\mathrm{mm})\end{array}$ & $\begin{array}{c}\text { Mean RNFL } \\
\text { measurement } \\
\text { error }(\mu m)( \pm S D)\end{array}$ & P-values \\
\hline \multirow[t]{7}{*}{ Average } & 0.1 & $2.3 \pm 2.0$ & \multirow{7}{*}{$<0.001$} \\
\hline & 0.2 & $3.4 \pm 3.1$ & \\
\hline & 0.3 & $4.5 \pm 4.5$ & \\
\hline & 0.4 & $5.8 \pm 5.9$ & \\
\hline & 0.5 & $7.9 \pm 7.5$ & \\
\hline & 0.6 & $10.1 \pm 9.1$ & \\
\hline & 0.7 & $12.1 \pm 11.4$ & \\
\hline \multirow[t]{7}{*}{ Superior } & 0.1 & $4.9 \pm 4.5$ & \multirow{7}{*}{$<0.001$} \\
\hline & 0.2 & $8.6 \pm 6.7$ & \\
\hline & 0.3 & $12.6 \pm 9.1$ & \\
\hline & 0.4 & $16.6 \pm 10.9$ & \\
\hline & 0.5 & $20.8 \pm 13.5$ & \\
\hline & 0.6 & $24.9 \pm 16.6$ & \\
\hline & 0.7 & $27.8 \pm 18.4$ & \\
\hline \multirow[t]{7}{*}{ Nasal } & 0.1 & $4.1 \pm 3.8$ & \multirow{7}{*}{$<0.001$} \\
\hline & 0.2 & $6.5 \pm 6.0$ & \\
\hline & 0.3 & $8.7 \pm 6.8$ & \\
\hline & 0.4 & $11.3 \pm 9.3$ & \\
\hline & 0.5 & $14.3 \pm 12.0$ & \\
\hline & 0.6 & $17.7 \pm 14.7$ & \\
\hline & 0.7 & $21.7 \pm 18.6$ & \\
\hline \multirow[t]{7}{*}{ Inferior } & 0.1 & $6.2 \pm 7.6$ & \multirow{7}{*}{$<0.001$} \\
\hline & 0.2 & $11.0 \pm 8.7$ & \\
\hline & 0.3 & $15.9 \pm 10.8$ & \\
\hline & 0.4 & $20.7 \pm 13.7$ & \\
\hline & 0.5 & $25.9 \pm 17.5$ & \\
\hline & 0.6 & $30.8 \pm 20.7$ & \\
\hline & 0.7 & $34.8 \pm 22.9$ & \\
\hline \multirow[t]{7}{*}{ Temporal } & 0.1 & $3.8 \pm 3.5$ & \multirow{7}{*}{$<0.001$} \\
\hline & 0.2 & $6.7 \pm 5.6$ & \\
\hline & 0.3 & $9.6 \pm 7.5$ & \\
\hline & 0.4 & $11.7 \pm 8.3$ & \\
\hline & 0.5 & $13.6 \pm 9.3$ & \\
\hline & 0.6 & $14.7 \pm 10.2$ & \\
\hline & 0.7 & $15.2 \pm 10.7$ & \\
\hline
\end{tabular}

Repeated-measure analysis of variance is used for the comparison of the mean RNFL measurement error at different scan displacement distances.
(512 A-scans) was 0.79 and the ICC for quadrant RNFL thicknesses ranged between 0.60 and 0.80 . An explanation for the lower RNFL thickness reproducibility during intersession measurement has been suggested to be related to scan placement variability. ${ }^{3}$ Consistent scan placement is more difficult to achieve for intersession measurement when the positions of head and eye have to be readjusted.

In agreement with the reported RNFL topologies, ${ }^{9-11}$ the derived RNFL models demonstrated that RNFL is thickest at superotemporal and inferotemporal sectors of the optic disc. The RNFL thickness gradually decreases when the nerve fibres course towards the macula and the peripheral retina. Therefore, it is not surprising that the superior and inferior RNFL measurements were most affected when the scan was displaced at the superior and the inferior directions (Figure 1c and e). Upon $0.7 \mathrm{~mm}$ scan displacement, the superior and inferior measurement errors could be as high as 28 and $35 \mu \mathrm{m}$, respectively. The average RNFL thickness, in contrast, showed the least change with different distances and directions of displacement. It is because when the scan is displaced superiorly, for example, an increase in the inferior RNFL thickness (because of the thicker RNFL towards the disc) always couples with a corresponding decrease in the superior RNFL thickness (because of thinner RNFL away from the disc) and thus resulting in a only small change in the average RNFL thickness. The mean average RNFL measurement error secondary to scan displacement ranged from $2.3 \pm 2.0 \mu \mathrm{m}$ for $0.1 \mathrm{~mm}$ scan displacement to $12.1 \pm 11.4 \mu \mathrm{m}$ for $0.7 \mathrm{~mm}$ scan displacement. This information could be useful to determine the limits of measurement variability during serial analysis of RNFL thickness. For example, the threshold for detecting changes at the superior or inferior RNFL measurements would need to be set higher than that for the average RNFL measurement. There are other factors that could be related to the variability of RNFL measurements. In a recent study, $\mathrm{Wu}$ et al $^{12}$ reported that variability in RNFL measurement was correlated with signal strength and analysis confidence although the effect of scan displacement was not examined.

Understanding the contributions of individual factors in determining the variability of RNFL measurement is important during longitudinal analysis to verify if progression occurs.

It is worth noting that the current model was developed based on an assumption that each scan was well-centred at the optic disc. However, it is difficult to validate whether this assumption was met because there is no objective criteria available in the Stratus OCT to confirm the position of the scan. Nevertheless, the selection of subjects with good fixation and the use of 'repeat mode' in keeping the position of internal fixation 
constant for each scan could have minimized the chance of obtaining off-centre scans. In addition, the observation that the modelled RNFL profiles closely matched with those generated manually in Stratus OCT (Figure 1b-e) suggests that the derived model is useful to predict and estimate the effect of scan circle displacement on the RNFL profiles. Although further investigation is required to validate the accuracy and reliability of the measurements obtained from the model, our findings have demonstrated the importance of scan centration for RNFL measurement in OCT. With the availability of the spectral domain OCT, it is possible to measure the RNFL topology more accurately and reliably by virtue of its faster scan rate and higher scan resolution. However, at the time of writing, software for RNFL measurements has not yet been available in most of the commercially available spectral domain OCT and none of them have been evaluated for RNFL measurement repeatability and reproducibility. It is also uncertain whether RNFL measurements obtained from spectral domain OCT can be used interchangeably with those from time domain OCT.

In summary, with the assumption that each concentric circle scan was well-centred in the cubic spline models, we observed that RNFL measurement could change substantially when the scan circle was displaced and these changes were related to the distance and direction of displacement. Obtaining well-centred scan is critical for reliable RNFL measurement.

\section{References}

1 Budenz DL, Chang RT, Huang X, Knighton RW, Tielsch JM. Reproducibility of retinal nerve fiber thickness measurements using the stratus OCT in normal and glaucomatous eyes. Invest Ophthalmol Vis Sci 2005; 46: 2440-2443.

2 Gurses-Ozden R, Teng C, Vessani R, Zafar S, Liebmann JM, Ritch R. Macular and retinal nerve fiber layer thickness measurement reproducibility using optical coherence tomography (OCT-3). J Glaucoma 2004; 13: 238-244.

3 Paunescu LA, Schuman JS, Price LL, Stark PC, Beaton S, Ishikawa $\mathrm{H}$ et al. Reproducibility of nerve fiber thickness, macular thickness, and optic nerve head measurements using StratusOCT. Invest Ophthalmol Vis Sci 2004; 45: 1716-1724.

4 Leung CK, Chan WM, Yung WH, Ng AC, Woo J, Tsang MK et al. Comparison of macular and peripapillary measurements for the detection of glaucoma: an optical coherence tomography study. Ophthalmology 2005; 112: 391-400.

5 Leung CK, Chan WM, Chong KK, Yung WH, Tang KT, Woo J et al. Comparative study of retinal nerve fiber layer measurement by Stratus OCT and GDx VCC, I: correlation analysis in glaucoma. Invest Ophthalmol Vis Sci 2005; 46: 3214-3220.

6 Medeiros FA, Zangwill LM, Bowd C, Vessani RM, Susanna Jr R, Weinreb RN. Evaluation of retinal nerve fiber layer, optic nerve head, and macular thickness measurements for glaucoma detection using optical coherence tomography. Am J Ophthalmol 2005; 139: 44-55.

7 Wollstein G, Schuman JS, Price LL, Aydin A, Beaton SA, Stark PC et al. Optical coherence tomography (OCT) macular and peripapillary retinal nerve fiber layer measurements and automated visual fields. Am J Ophthalmol 2004; 138: 218-225.

8 De Boor C. A Practical Guide to Splines. Rev. Ed Springer: New York, 2001

9 Dichtl A, Jonas JB, Naumann GO. Retinal nerve fiber layer thickness in human eyes. Graefes Arch Clin Exp Ophthalmol 1999; 237: 474-479.

10 Varma R, Skaf M, Barron E. Retinal nerve fiber layer thickness in normal human eyes. Ophthalmology 1996; 103: 2114-2119.

11 Jonas JB, Nguyen NX, Naumann GO. The retinal nerve fiber layer in normal eyes. Ophthalmology 1989; 96: 627-632.

12 Wu Z, Vazeen M, Varma R, Chopra V, Walsh AC, LaBree LD et al. Factors associated with variability in retinal nerve fiber layer thickness measurements obtained by optical coherence tomography. Ophthalmology 2007; 114: 1505-1512. 